Subscriber access provided by WEBSTER UNIV

Article

\title{
Direct coupling of dispersive extractions with magnetic particles to mass spectrometry via microfluidic open interface
}

Marcos Tascon, Varoon Singh, Mohammad Huq, and Janusz Pawliszyn

Anal. Chem., Just Accepted Manuscript • DOI: 10.1021/acs.analchem.9b00308 • Publication Date (Web): 05 Mar 2019

Downloaded from http://pubs.acs.org on March 5, 2019

\section{Just Accepted}

"Just Accepted" manuscripts have been peer-reviewed and accepted for publication. They are posted online prior to technical editing, formatting for publication and author proofing. The American Chemical Society provides "Just Accepted" as a service to the research community to expedite the dissemination of scientific material as soon as possible after acceptance. "Just Accepted" manuscripts appear in full in PDF format accompanied by an HTML abstract. "Just Accepted" manuscripts have been fully peer reviewed, but should not be considered the official version of record. They are citable by the Digital Object Identifier (DOI®). "Just Accepted" is an optional service offered to authors. Therefore, the "Just Accepted" Web site may not include all articles that will be published in the journal. After a manuscript is technically edited and formatted, it will be removed from the "Just Accepted" Web site and published as an ASAP article. Note that technical editing may introduce minor changes to the manuscript text and/or graphics which could affect content, and all legal disclaimers and ethical guidelines that apply to the journal pertain. ACS cannot be held responsible for errors or consequences arising from the use of information contained in these "Just Accepted" manuscripts. 


\title{
Direct coupling of dispersive extractions with magnetic particles to mass spectrometry via microfluidic open interface
}

\author{
Marcos Tascon ${ }^{\dagger}, ¥$, Varoon Singh ${ }^{\dagger}$, Mohammad Huq, Janusz Pawliszyn* \\ Department of Chemistry, University of Waterloo, Waterloo, Ontario N2L 3G1, Canada \\ *Email janusz@uwaterloo.ca, Fax: 519-746-0435; Tel: 519-888-4567
}

\begin{abstract}
Microextraction coupled to MS has great potential in analytical chemistry laboratories operating in a variety of fields. Indeed, microextraction methods directly coupled to MS can be of large value given that they can provide not only analyte extraction and enrichment, but also effective sample cleanup. In recent years, the practicality in handling, high active surface area, versatility, and environmentally friendly features of magnetic dispersive microextraction technologies have contributed to an explosion in the number of methods and technologies reported in the literature for a wide range of applications. However, to the best of our knowledge, no technology to date has been capable of efficiently merging these two rising concepts in a simple and integrated analytical workflow. In this context, the microfluidic open interface is presented for the direct coupling of dispersive magnetic extraction to mass spectrometry. This technology operates under the concept of a flow-isolated desorption volume, which generates a stagnant droplet open to ambient while continuously feeding with solvent the ionization source by means of the self-aspiration process intrinsic of the ESI interface. To improve the efficiency of the final analytical workflow, a novel dispersive magnetic micro- and nanoparticle extraction protocol for biofluid droplets was developed. The final methodology entailed the dispersion of a small amount of magnetic particles (20-70 $\mu \mathrm{g})$ in a droplet of biofluid $(\leq 40 \mu \mathrm{L})$ for extraction, followed by a particle collection step using a home-made 3D-printed holder containing an embedded rare-earth magnet. In the final step, the holder is set on top of the MOI for desorption in the isolated droplet. By switching the valve, the desorbed analytes are transferred to the ESI source in less than $5 \mathrm{sec}$. As proof of concept, the completely new setup was applied to the determination of prohibited substances from PBS and human urine using $\mathrm{Fe}_{2} \mathrm{O}_{3}$ magnetic nanoparticles $\left(50 \mathrm{~nm}\right.$ ) functionalized with $\mathrm{C}_{18}$. The LOQs obtained were in the low-ppb range in all cases, and acceptable precision $(\leq 20 \%)$ and accuracy $(80-120 \%)$ were attained. Also, taking advantage of the fast extraction kinetics provided by the radial diffusion associated with small particles, the methodology was employed for the selective extraction of phosphopeptides from $40 \mu \mathrm{L}$ of tryptic $\beta$-casein digest using $70 \mu \mathrm{g}$ of magnetic Ti-IMAC microparticles. To conclude, the technology and methodology herein presented provided excellent capabilities comparable to other SPME-MS approaches while dramatically minimizing the amount of sample and sorbent required per analysis, as well as affording significantly fast extraction times due to the enhanced kinetics of extraction.
\end{abstract}

Propelled by the advances in mass spectrometry related to analyzers and ambient ionization techniques, ${ }^{1,2}$ the direct-to-MS analysis field has emerged to encompass a large collection of sensitive and extremely fast technologies. Among the various technologies introduced to date, desorption electrospray (DESI), ${ }^{3}$ direct analysis in real time (DART),${ }^{4}$ and solid-substrate electrospray, ${ }^{5,6}$ stand as some of the most widespread techniques, with several reported applications in clinical, ${ }^{7,8}$ environmental, ${ }^{9,10}$ and forensic sciences. ${ }^{11,12}$ However, analysis of complex matrices via direct ionization and introduction to mass spectrometers can evidence several drawbacks, such as ion suppression and poor limits of detection, which limit the quality of data that can be attained by such methods, as well as frequent mass spectrometer contamination, which leads to increased costs and delays associated with instrument maintenance. Smart and efficient sample preparation strategies that circumvent the above issues without sacrificing total analysis time and simplicity are thus highly desirable, if not central, to the implementation of direct-to-MS applications involving complex matrices. This concept, representing a vital step in the transfer of directto-MS techniques to clinical and point-of-care applications, has spurred the development of several sample preparation approaches in recent years. For instance, the development and subsequent application of slug-flow liquid-liquid microextraction inside a nano-ESI emitter for extraction of prohibited substances from different biofluids was shown to achieve acceptable limits of detection and preconcentration factors. ${ }^{13}$ In the solid-liquid microextraction area, several technologies for direct coupling, such as liquid extraction surface analysis
(LESA), ${ }^{14,15}$ open port sample interface (OPSI), ${ }^{16-18}$ and the capillary gap sampler, ${ }^{19}$ have been reported. As one of their highlights, the above techniques are well-known for their superior speed of analysis, enabling times of analysis per sample of just a few seconds. ${ }^{16,17}$ Although these technologies extract using the same concept, extraction time in OPSI and the capillary gap sampler cannot be optimized due to the dynamic nature of these technologies, which operate via a constant flow of solvent towards the mass spectrometer. Notable direct-to-MS technologies using sample preparation steps such as on-line solid-phase extraction (SPE-MS) as well as solid-phase microextraction (SPME-MS) have also been introduced recently. However, while both techniques involve a solid extractive phase in contact with the sample, the fundamentals of extraction utilized by these two seemingly similar techniques are decidedly different. Succinctly, SPME is a non-exhaustive extraction technique driven by diffusion and equilibrium distribution between phases whereas SPE operates under exhaustive extraction conditions. ${ }^{20}$ The attributes of the former endow SPME with a level of flexibility in design, allowing for multiple configurations beyond the classical fibers. ${ }^{21}$ As for SPE-based techniques, current SPEMS options, such as RapidFire ${ }^{\circledR 22}$ and Turboflow ${ }^{\circledR},{ }^{23}$ consist of an SPE cartridge hyphenated on-line to MS via ESI, and a highly pressurized system that enables fast flushing of samples, washing solvent, and desorption solution. Although several applications have demonstrated analysis times below 20 seconds and low limits of quantitation, the high chance of system clogging limits the compatibility of these systems to only certain biomatrices. Certainly, biological samples such as plasma and 
whole blood cannot be processed by these systems without incorporation of additional sample preparation steps into the workflow. As for SPME-based applications, a wide variety of technologies, employing different geometries and ionization methods, have been developed for the direct coupling of SPME to MS. ${ }^{24-27}$ Geometries such as blades, ${ }^{28}$ meshes, ${ }^{29}$ tubes,${ }^{26}$ and fibers ${ }^{27}$ have been successfully adopted in different applications. ${ }^{24-29}$ However, the majority of said SPME-MS dispositives developed to date are limited to configurations where fixed sorbents are deposited onto a substrate. The use of such configurations severely limits the active surface area of said probes, a factor which in turn limits the recovery amounts of target analytes. In this context, dispersive SPME has emerged as a valuable option, given that this technique utilizes particles for extraction of analytes. In dispersive SPME, functionalized particles are directly dispersed into the sample matrix, thus maximizing the active surface area of the substrate while also increasing the mass transfer rate of analytes due to radial diffusion, a feature that is enabled by the size of the particles as well as the thin coatings. ${ }^{30}$ Furthermore, the absence of a gluing media, which enables direct contact between analytes and the extractive phase, contributes to increasing mass transfer coefficients, thus dramatically decreasing extraction times. ${ }^{31,32}$ Therefore, efficient and smart direct hyphenation of dispersive SPME to mass spectrometry can offer a variety of benefits, such as speed of analysis, reduction in sample consumption, and increased sensitivity. However, in order to successfully achieve this goal, particles must be handled in such way so as to minimize and simplify the number of steps involved, as well as reduce the overall time of analysis. While classical particle handling methods such as centrifugation or filtration, which are time consuming and hard to automate, are often adopted within the dispersive sample preparation workflow, use of magnetic micro- and nanoparticles enables manipulation of the extractive phase via a magnetic field, a process that highly simplifies the workflow of analysis. ${ }^{33,34}$ First attempt was developed by Chen et al., ${ }^{35}$ where a classical magnetic dispersive-SPE procedure was carried out. Then, collected magnetic nanoparticles were directly exposed to a desorption corona beam ionization source with MS detection. The currently presented work introduces a technology that directly couples dispersive-SPME to mass spectrometry through the use of magnetic micro- and nanoparticles as extraction phase. The developed interface is a novel variation of the previously reported microfluidic open interface (MOI). ${ }^{27}$ Although MOI technology also works under the concept of an isolated desorption volume, ${ }^{27}$ in this case, instead of a microfluidic desorption chamber, the interface is consisted of an isolated droplet $(\approx 3-5 \mu \mathrm{L})$ connected to the ESI source via a modified open port probe (OPP) developed by Van Berkel et al. ${ }^{36,37}$ In this analytical workflow, extraction is carried out via dispersion of magnetic particles (15-100 $\mu \mathrm{g} / \mathrm{sample})$ in a droplet of sample $(\leq 50 \mu \mathrm{L})$. Next, a magnetic field is applied for particle collection, followed by gentle rinsing of the collected particles so as to remove any unspecific matrix attachments such as macromolecules and salts from their surface. Finally, the isolated droplet of the MOI is put in contact with the collected spot of particles in order to desorb the targeted analytes $(\leq 10 \mathrm{sec})$ and quickly transfer them to the MS via ESI ionization. As a proof of concept, extractions of prohibited substances from droplets of phosphate buffer saline and pooled urine were carried out using iron oxide nanoparticles functionalized with $\mathrm{C}_{18}$. Extractions were carried out for $30 \mathrm{~s}$, whereupon particles were collected, rinsed, and desorbed in the interface for a period briefer than $10 \mathrm{~s}$. All compounds were detected within a range between
$0.5 \mathrm{ng} \cdot \mathrm{mL}^{-1}$ and $5 \mathrm{ng} \cdot \mathrm{mL}^{-1}$ for both matrices, with an overall time of analysis below 2 min per sample. Selective extraction of phosphopeptides from a $\beta$-casein digest using a magnetic TiIMAC microparticles was also carried out as part of this work. Full scan acquisition mode evidenced recovery of this high molecular weight phosphopeptides, with an overall time of analysis below 20 minutes. The herein presented technology attained figures of merit comparable to other direct-to-MS approaches $^{13,28,37,38}$ and classical off-line strategies, while using 10 to 100 times less extractive material and enabling significantly faster analysis times. ${ }^{39}$

\section{EXPERIMENTAL SECTION}

Materials and Supplies Formic acid (FA), ammonium acetate (both LC-MS grade), $\beta$-casein (BioUltra, $\geq 98 \%$, C6905), TPCK modified sequencing grade trypsin (11418 475001$)$, 1,4-dithiotheritol, iodoacetamide (BioUltra, I1149), ammonium bicarbonate $\left(\mathrm{NH}_{4} \mathrm{HCO}_{3}\right)$, polyacrylonitrile (PAN), and ethanol (reagent grade) were all purchased from Sigma-Aldrich (Oakville, ON, Canada). LC-MS grade methanol $(\mathrm{MeOH})$ and water as well as urea were purchased from Fisher Scientific (Ottawa, Canada). MagReSyn ${ }^{\circledR}$ Ti-IMAC magnetic microparticles was purchased from Phenoswitch Bioscience Inc. (Sherbrooke, QC, Canada). Cocaine, cocaine $\mathrm{d}_{3}$, fentanyl, fentanyl $\mathrm{d}_{5}$, methadone, methadone $\mathrm{d}_{3}$, propranolol, propranolol $\mathrm{d}_{7}$, sertraline, and sertraline $\mathrm{d}_{3}$ were acquired from Cerilliant Corporation (Round Rock, TX, USA). $\mathrm{Fe}_{2} \mathrm{O}_{3}$ nanoparticles ( $\geq 50 \mathrm{~nm}$ ), Chloro(dimethyl)octadecylsilane, and triethylamine were purchased from MilliporeSigma (Milwaukee, WI, USA) and used as received. A detailed description of the functionalized magnetic nanoparticle synthesis and their characterization can be found in Supporting Information (section 1). A phosphate-buffered saline solution (PBS) ( $\mathrm{pH} 7.4$ ) was prepared according to the procedure outlined in Supporting Information (section 2). For small molecules, stock standard solutions were prepared in methanol at a concentration of $1000 \mu \mathrm{g} \cdot \mathrm{mL}^{-1}$ and stored at $-80{ }^{\circ} \mathrm{C}$. The urine collection procedure carried out as part of this study was conducted with the approval of the University of Waterloo's Office of Research Ethical Board. Tryptic digestion of $\beta$-casein was followed by a standard in-solution digestion protocol with some modifications. This procedure is described in detail in Supporting Information (section 3).

Interface setup The interface of the developed device consists of two main sections. As shown in figure $1 \mathrm{~A}$, the main piece has a lower hole of $3.18 \mathrm{~mm}$ diameter that connects it to the upper part of the apparatus via a channel with a diameter of 0.5 $\mathrm{mm}$ and a length of $2 \mathrm{~mm}$. Aiming to avoid potential carry-over between samples, the tip of the dispositive was designed with a tapered end that reduces the wetting surface of the device at the moment a droplet comes in contact with the sample. The upper $10 \mathrm{~mm}$ of the device has a reduced external outer diameter of 4 $\mathrm{mm}$ to avoid droplet spread by capillarity when the device comes in contact with the well's flat surface. The connection between the top piece and the ESI source was inspired by the design of the open-port interface reported by Van Berkel et al. ${ }^{36,37}$ Essentially, the interface is comprised of two concentric tubes where solvent is delivered by a pump through a tee junction, where it fills the gap formed between these two tubes. Once the solvent reaches the top of the interface, it is aspirated by the inner tube towards the mass spectrometer via the Venturi effect generated at the ESI source. ${ }^{36}$ The outer tube is comprised of a 304 stainless steel tube with dimensions $1.75 \mathrm{~mm}$ i.d. $\times$ 
$3.18 \mathrm{~mm}$ o.d. $\times \sim 5 \mathrm{~cm}$ long (McMaster-Carr, Chicago, IL, USA) and the inner tube is a peek tube; $180 \mu \mathrm{m}$ i.d. $\mathrm{x} 0.75 \mathrm{~mm}$ o.d. $\times$ $\sim 20 \mathrm{~cm}$ long (Idex, Oak Harbor, WA, USA) embedded inside of a $0.75 \mathrm{~mm}$ i.d. x $1.52 \mathrm{~mm}$ o.d.x $15 \mathrm{~cm}$ long FEP tubing (ColeParmer, Vernon Hills, IL, USA). Solvent is delivered by an LC pump (200 Series; Perkin Elmer, Santa Clara, CA, USA) and the ESI and aspiration are generated by a TurboIon spray source (Sciex, Concorde, ON, Canada). To increase the aspiration rate, the ESI commercial electrode was substituted with a $150 \mu \mathrm{m}$ i.d. electrode (McMaster-Carr, Chicago, IL, USA). The fluidic system was connected by means of a PEEK Tee junction (Idex, Oak Harbor, WA, USA). As illustrated in Fig. 1, the pumping solvent was bypassed with a 6-port valve in order to rapidly switch the pump flow, allowing for rapid aspiration of the isolated droplet towards the MS. ${ }^{27}$ The operational conditions for the desorption step are: pump flow of $100 \mu \mathrm{L} \cdot \mathrm{min}^{-1}$, positive ionization mode, nitrogen gases set at GS1 = 90, GS2 = 70; curtain gas $=25$; heated nebulizer temperature $=300{ }^{\circ} \mathrm{C}$; and electrospray voltage $=5500 \mathrm{~V}$. Regarding the second section of the device, Figure 1B shows the upper holder that enables physical contact between the droplet and the collected magnetic particles. The upper holder contains a $5 \mathrm{~mm}$ diameter $\mathrm{x} 5 \mathrm{~mm}$ long rare-earth magnet (Lee-Valley, ON, Canada) that remains in contact with the plate, keeping the particles attached to its surface. The holder was 3D printed with Nylon and sits perfectly on top of the modified MOI. The plates are made of $2 \mathrm{~cm} \mathrm{x} 2$ $\mathrm{cm}$ Teflon ${ }^{\circledR}$ squares with a thickness of $5 \mathrm{~mm}$ containing a $2 \mathrm{~mm}$-deep well with a diameter of $4 \mathrm{~mm}$. Once extraction is performed, the plate is easily slid into the holder. Figure $1 \mathrm{C}$ illustrates how the holder containing the well can be easily positioned on top of the MOI, allowing for contact to occur between the isolated droplet and the magnetic particles.

Analysis of prohibited substances PBS and urine samples were spiked with concentrations of cocaine, fentanyl, methadone, propranolol, and sertraline ranging between 0.1 and 100 $\mathrm{ng} \cdot \mathrm{mL}^{-1}$. All employed internal standards (see Table S1) were spiked at $10 \mathrm{ng} \cdot \mathrm{mL}^{-1}$. Samples were agitated and stored for three hours so as to allow for equilibration between analytes and matrix components to occur. Prior to extraction, an aliquot consisted of $20 \mu \mathrm{L}$ of a slurry of $\mathrm{Fe}_{2} \mathrm{O}_{3}-\mathrm{C}_{18}$ nanoparticles $3 \mathrm{mg} \cdot \mathrm{mL}$ ${ }^{1}(60 \mu \mathrm{g}) 50: 50 \mathrm{v} / \mathrm{v}$ (ether/methanol) was added to the Teflon well. Contents were left to rest for a few seconds prior to extraction so as to allow the solvent in the slurry to evaporate from the mixture. Then, $40 \mu \mathrm{L}$ of sample (PBS or urine) was added to the well. Next, a sonication probe was utilized in order to disperse the particles into the droplet. Essentially, a stainlesssteel wire attached to the tip of the probe allowed for efficient transference of ultrasound waves transmitted from the probe to the droplet. Following dispersion, the probe was rinsed with methanol for $5 \mathrm{~s}$ so as to avoid any kind of carryover. Particles were kept dispersed for $30 \mathrm{~s}$ so as to allow for extraction to occur. The extraction procedure was completed by applying a magnetic field to collect the particles. Once the sample was discarded, the particles were rinsed with $400 \mu \mathrm{L}$ of water. Immediately following, the well was slid in the top holder, and put in contact with the isolated droplet of the MOI for $5 \mathrm{~s}$. After desorption, the valve was switched, allowing for rapid introduction of the desorption solution into the MS. MRM transitions are detailed in table $S 1$.

Analysis of phosphopeptides Phosphopeptides were extracted from a $\beta$-casein digest (see Supporting Information, section 3) using Ti-IMAC magnetic microparticles (MagReSyn ${ }^{\circledR}$ TiIMAC). Particles were washed and equilibrated according to manufacturing specifications. In brief, particles were separated from the shipping solution by centrifugation and removal of the supernatant. Next, particles were resuspended and washed in $70 \%$ ethanol with gentle agitation (i.e. vortex mixing) for $5 \mathrm{~min}$.

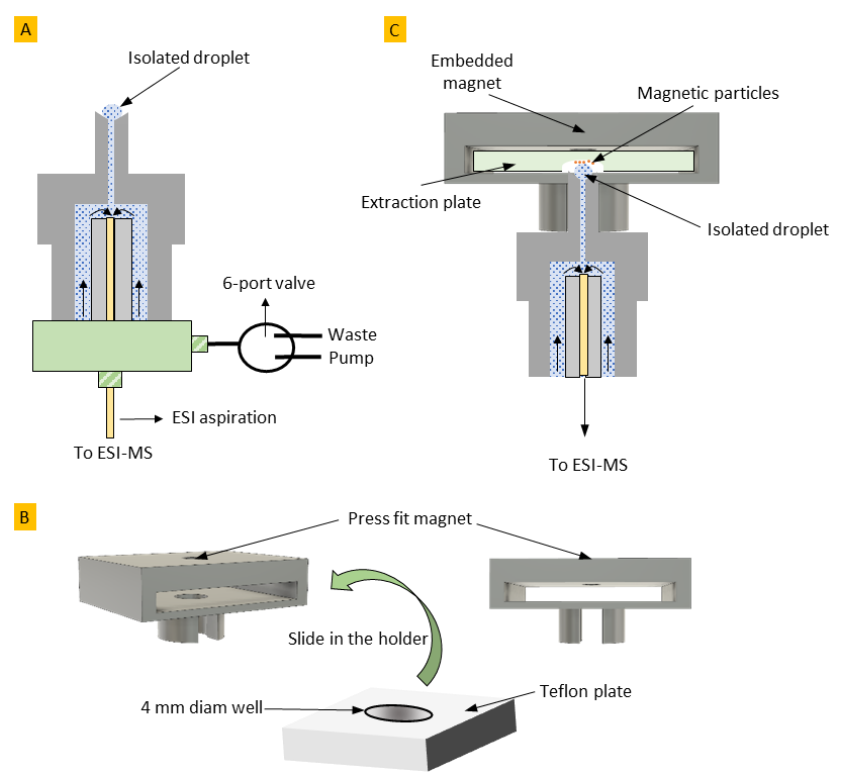

Figure 1 Schematics of A- modified MOI with isolated droplet as desorption volume, B- 3-D printed magnetic holder with well plate and, C- Desorption setup fitting the holder with the MOI. The tube containing the particles was then placed on a magnetic separator so as to allow microparticles to decant. The ethanolic solution was next removed by aspiration with a pipette and discarded. This washing procedure was repeated twice. Next, particles were submitted to a basic washing step, using $100 \mu \mathrm{l}$ of $1 \% \mathrm{NH}_{4} \mathrm{OH}$ with gentle agitation (i.e. vortex mixing) for 10 min. Following, particles were magnetically collected, and the solution removed. Finally, the particles were equilibrated with1M glycolic acid in $80 \%$ ACN, 5\% TFA for 60 seconds. After removing the solution, a suspension of $3 \mathrm{mg} \cdot \mathrm{mL}^{-1}$ of microparticles was prepared in 50:50 v/v (ether/methanol). A volume corresponding to $70 \mu \mathrm{g}$ of microparticles was deposited onto the well. After allowing for a few seconds to elapse so as to ensure evaporation of solvent, a droplet of $40 \mu \mathrm{L}$ of $\beta$-casein digest was added in the well, followed by $5 \mathrm{~s}$ of sonication using the $\mu$-sonication probe. Extraction was allowed to take place for a period of $15 \mathrm{~min}$, after which a magnetic field was applied to collect the particles, whereupon the sample was discarded. The particles were rinsed with $400 \mu \mathrm{L}$ of a solution consisted of $0.1 \% \mathrm{NH}_{4} \mathrm{OH} 80: 20 \mathrm{v} / \mathrm{v}$ (ACN:water). The well was slid in the holder and positioned on top of the MOI for $10 \mathrm{~s}$ so as to allow for desorption to take place. The MOI solvent used was methanol $0.1 \% \mathrm{NH}_{4} \mathrm{OH}$, and operational conditions were as stated in the previous section. After desorption, the valve was switched for 6 seconds so as to allow for introduction of the droplet into the MS. Instrumental detection was carried out in full scan mode within an $\mathrm{m} / \mathrm{z}$ range between 600 and 1800 , with a scan time of $500 \mathrm{~ms}$. MOI conditions were the same than in previous section.

\section{RESULTS AND DISCUSSION}

Dispersive microextraction in droplets using magnetic particles In order to perform efficient hyphenation between sample preparation and MS, a wide range of variables, from both the chemical and the technological perspectives, must be considered. 


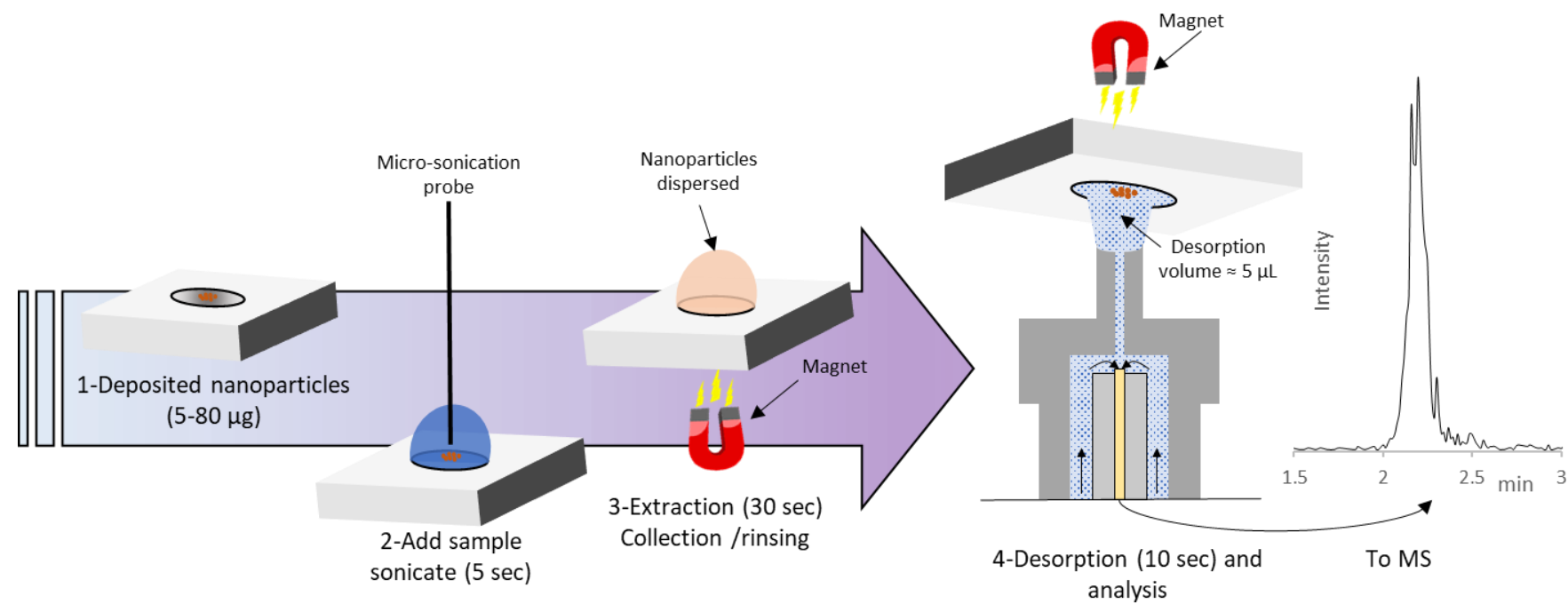

Figure 2 Analytical workflow of droplet magnetic dispersive extraction coupled to MS via MOI.

Key desirable characteristics included fast handling of particles, process reproducibility, and efficient transference of analytes from sorbent to MS are considered. Hence, the way the extraction step is performed plays an important role in the general analytical workflow. The first stage of method development consisted of the design of a strategy to disperse the magnetic particles inside the droplet. For this purpose, a stainless-steel wire was adapted to a sonication probe, allowing for transmission of ultrasound waves through the micro-probe and precise delivery into the droplet. This enabled dispersion of either micro or nanoparticles to take place in less than three seconds. As video S1 shows, once the micro-probe is in contact with the sample droplet, a negligible amount of energy is enough to proficiently disperse and homogeneously distribute the particles inside the droplet. During the extraction process, the amount of time required for extraction, during which particles must remain dispersed, is dependent on particle size, the chemistry of the particle surface, the chemical nature of the well and the nature of the sample. For instance, when handling aqueous-based samples, dispersion of $50 \mathrm{~nm} \mathrm{Fe}_{2} \mathrm{O}_{3}-\mathrm{C}_{18}$ particles allowed for a total time of two hours of adequate dispersion of particles into the matrix. As can be seen in figure S3(A-G) the only limiting factor in this case was the evaporation rate of the sample. Time of dispersal was also evaluated while dispersing Ti-IMAC magnetic microparticles. Maximum dispersion times were approximately 60 min for this particle size $(5-10 \mu \mathrm{m})$. This experimental trend indicates that the bigger the particles the shorter the time particles can be dispersed in a droplet by simply sonication. Thus, it can be inferred that gravity plays a significant role in shortening dispersion times. In cases where longer extraction times are needed, either the use of a second sonication or addition of a chemical stabilizer may be valid strategies to maintain dispersion. Keeping this in mind, particle collection represents another critical step that must be optimized for high-throughput analysis. In this step, the use of permanent rare-earth magnets yielded better results than electromagnets despite the higher practicality of the latter due to the magnitude of the magnetic field applied. For this purpose, a holder with an embedded magnet was specifically designed and 3D printed (figure 1B). Hence, once extraction is concluded, the Teflon ${ }^{\circledR}$ plate is slid in the holder and put in close contact with the magnet. Since the largest distance needed for a particle to travel is the diameter of the droplet $(\approx 2 \mathrm{~mm})$, all particles are collected in less than $10 \mathrm{~s}$ (Video S2). At this point, considering that the desorption step will consist of putting into contact a solvent with the collected particles, followed by direct injection of this liquid, a critical point to evaluate is how well these particles are retained in the presence of a rinsing fluid. Figure $S 4$ displays micrographs of the rinsing solution used after extraction with $120 \mu \mathrm{g}$ of nanoparticles (Figures $S 4 A-B$ ) and $20 \mu \mathrm{g}$ of nanoparticles (Figures $S 4 C-D)$. As can be garnered from the images, $120 \mu \mathrm{g}$ of particles sets the upper limit of particles that the system can tolerate, as the results of the micrographs of the rinsing step show the presence of some remains due to the loose of efficiency in the collection step. While the subsequent desorption step did not present particles in the solution, since these were removed during the washing step, higher amounts of particles would likely provoke losses in reproducibility. On the other hand, figures S4 $\mathrm{C}$ and S4 D represent conditions where no displacement of nanoparticles occurred at all. This limit was experimentally found to be below $100 \mu \mathrm{g}$ of nanoparticles. In the case of the magnetic microparticles, the handling of the particles was found to be simpler than that of nanoparticles since the influence of gravity aided in the collection and detainment. In summary, most of the technological challenges discussed in this section regarding the dispersion, collection, and handling of particles were overcome by completely new alternatives to traditional dispersive extraction techniques. Further, this work introduces a new extractive technology that efficiently fits into a high-throughput analytical workflow.

Analytical workflow The methodology herein presented is integrated by tailoring each of the steps for compatibility, from the extraction stage to MS analysis. This will undoubtedly facilitate automation given the level of simplicity and short number of steps that compose the workflow. Figure 2 shows the schematics of the analytical workflow. At the first stage, the particles are deposited on the well plate using a slurry of a volatile solvent or mixture of solvents as a vehicle. In terms of weight, a weight range of particles within $3 \mu \mathrm{g}$ and $100 \mu \mathrm{g}$ was found to still allow for proper handling while circumventing losses of particles during the rinsing and desorption steps. Once the slurry is evaporated, a droplet of sample $(10-50 \mu \mathrm{L})$ is added. Then, the sonication microprobe is inserted in the sample droplet, followed by 5 seconds of sonication to homogeneously disperse the particles (step 2). Dispersion is maintained during this step so as to allow for static extraction to occur. Once the extraction step is concluded, the well plate is slid in the holder, where the magnet collects all magnetic particles. 

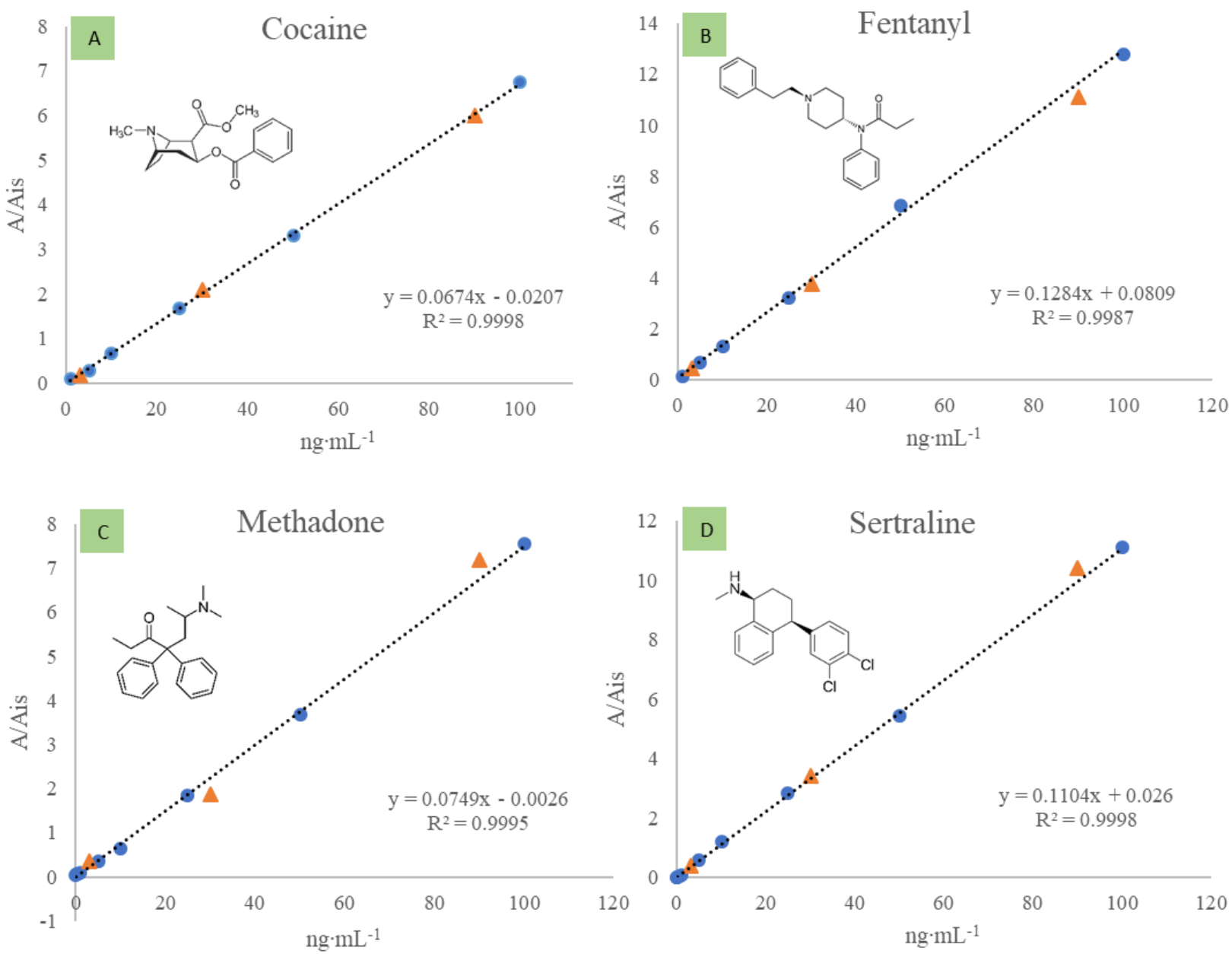

Figure 3 Calibration curves for determination of A) Cocaine, B) Fentanyl, C) Methadone and D) Sertraline from urine. Blue squares represent calibration points while orange triangles represent validation points.

The sample is then discarded, and particles are rinsed with 400 $\mu \mathrm{L}$ of a water/solvent mixture using a pipette (step 3). Finally, in step 4, the holder is flipped and positioned on top of the modified MOI. At this point, the isolated droplet is in contact with the particles, generating a liquid junction between the MOI and the surface of the particles produced by the solvent surface tension and viscosity (see Figure 2). Since the isolated droplet volume is practically constant, the contact geometry does not change during the time needed for desorption to occur, thus allowing for a reproducible desorption process. Theoretically, the desorption volume of the isolated droplet does not exceed $3 \mu \mathrm{L}$; thus, a great enrichment factor is also attained in this manner. The applications developed as part of this work yielded preconcentration factors of about 15 -fold, since sample volumes were $40 \mu \mathrm{L}$, and recoveries close to $100 \%$. Once the desorption stage, which lasts approximately 10 seconds, is concluded, the valve is then switched in order to bypass the pump flow and aspirate the desorbed analytes, transfer them towards the ESI, then finally to the MS. As mentioned in our previous work, ${ }^{27}$ the desorption time of the developed technology can be optimized. Maximum practical values were close to 30 seconds, where no changes in droplet shape and volume were observed. As can be seen in Figure 2, the peak has a band width of about 20 s, giving an approximate width at half-height of $5 \mathrm{~s}$. Undoubtedly, the aforementioned features render the method not only as fast and high-throughput compatible, but also as highly sensitive, providing high enrichment factors while minimizing analyte losses.
Determination of prohibited substances from biofluids As a proof of concept, the developed methodology was applied for detection and quantitation of a group of prohibited substances present at low ppb levels in PBS and human urine. Figure S3 shows calibration curves for cocaine, fentanyl, methadone, and sertraline in PBS. As can be clearly seen, great linearity $\left(r^{2} \geq 0.99\right)$ and LOQs below $1 \mathrm{ng} \cdot \mathrm{mL}^{-1}$ were attained in all cases (see details in Table S2). Furthermore, the amount recovered is in agreement with established $\log \mathrm{P}$ values, demonstrating typical $\mathrm{C}_{18}$-based sorbent behavior (Table S1). The accuracy values at three different validation points $\left(3,30\right.$ and $\left.90 \mathrm{ng} \cdot \mathrm{mL}^{-1}\right)$ were acceptable and within a range between 81 and $112 \%$, while precision values averaged at $10 \%$. As for urine determinations, calibration curves, including the three validation points, are shown in Figure 3. In general, the calibration plots yielded good linearity $\left(r^{2} \geq 0.99\right)$ as well as precision (average $9 \%$ ), with satisfactory accuracy within a range between 84 and $116 \%$ (see details in Table S3). Furthermore, LOQs only slightly lower than those attained for PBS samples, of values 5, 1, 5, 5 and 1 $\mathrm{ng} \cdot \mathrm{mL}^{-1}$ corresponding to cocaine, fentanyl, methadone, propranolol, and sertraline, respectively, were attained for urine samples. This fact indicates the presence of matrix effects most likely due to the presence of ion suppression in the ionization process. As a result, the sensitivity of the method decreases in comparison with the obtained in PBS. Nevertheless, it was found that the internal standards added were able to account for all the errors, making this method perfectly suited for this application. 


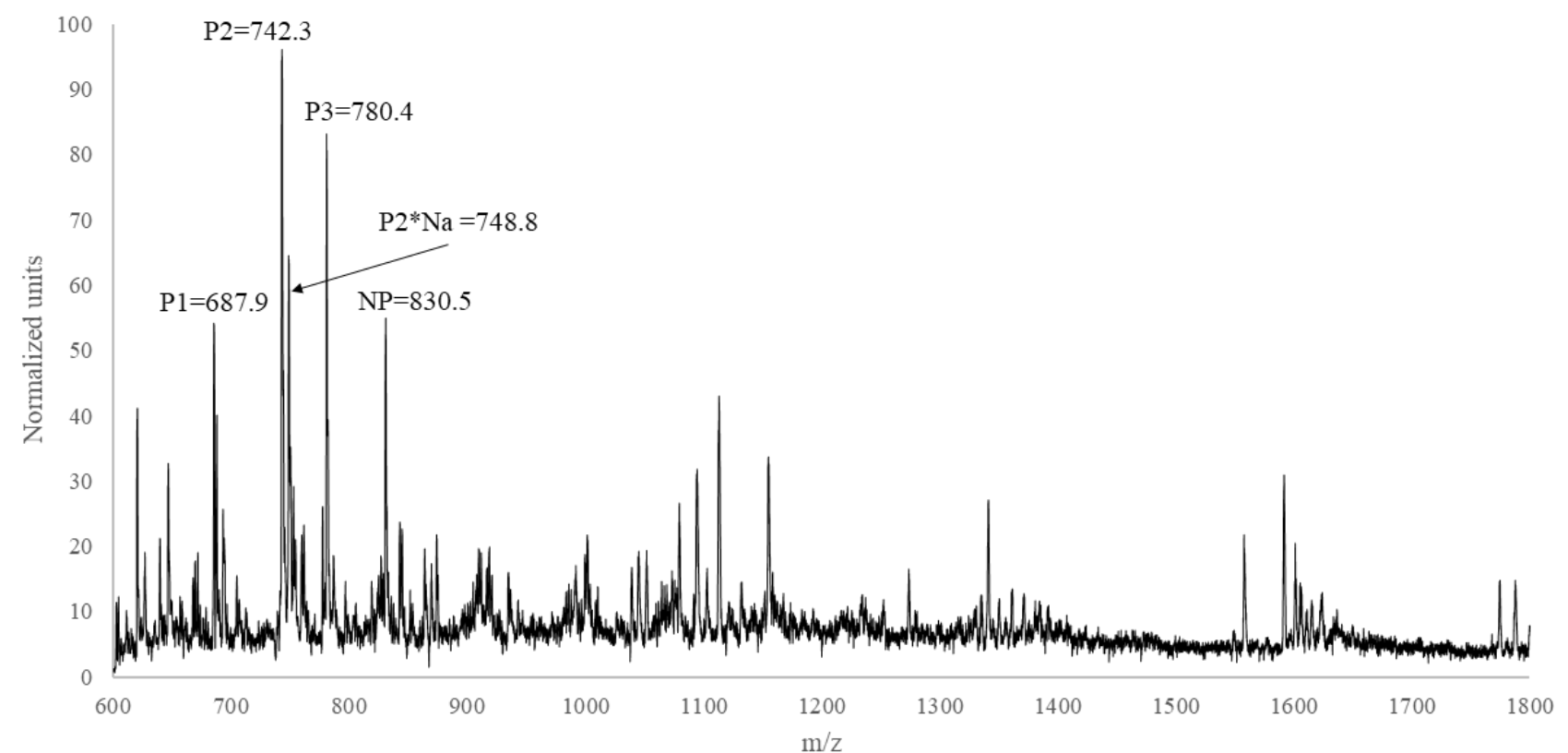

Figure 4 Full scan mass spectra of an extract from beta casein digest directly coupled to MS via MOI. The particles used in these experiments were Ti-IMAC magnetic microparticles.

Although the LOQs for urine were slightly poorer than those found for PBS, in all cases, minimum required performance levels (MRPL) cut-off values were met for all compounds (Table S1). Thus, the methodology herein developed agrees with all sensitivity, accuracy, and precision requirements of other ambient MS or SPME strategies. ${ }^{28,38,40,41}$ Sample consumption is very low, commonly in the range between $20-40 \mu \mathrm{L}$, which is comparable to previously reported approaches. ${ }^{38,40,42}$ However, the amount of sorbent employed is dramatically lower - at least 20 fold lower when compared with any other dispersive extraction or microextraction methodologies. ${ }^{39,43} \mathrm{It}$ is important to point out that the method not only minimizes the absolute amount of nanoparticles used, but also the ratio sorbent-sample amount, which is reduced approximately by a factor of 2 by taking advantage of SPME extraction fundamentals. The latter advantage also enables the attainment of extremely high enrichment factors on sorbent (average of 2500 times) and equilibration times of about $30 \mathrm{sec}$. While the final enrichment factor is technologically limited by the desorption volume, this development ensures at least a tenfold enrichment factor due to the ability of the method to enable desorption of target analytes in less than $5 \mu \mathrm{L}$, with recoveries at over $90 \%$. This fast kinetics of extraction and desorption can be explained by the radial diffusion contribution, which is owed to the size of the particles as well as the very thin coating of the extractive phase $(15 \mathrm{~nm}){ }^{21}$ Although the magnetic nanoparticles can be recovered and reused by simply washing and conditioning, the negligible amount of particles required for experiments make the particles practically disposable, since an average of only $10 \mathrm{mg}$ of nanoparticles are used for every 200 analyses.

Screening of phosphopeptides from protein digest Taking advantage of the enhanced kinetics of extraction demonstrated in the previous application, molecules within the $\mathrm{m} / \mathrm{z}$ range 500-1000 were also evaluated. These molecules, having a moderate to high affinity constant towards the extraction phase, usually take several hours to reach equilibrium. ${ }^{32}$ Therefore, in this section, the profiling of phosphopeptides from beta casein is used as a proof of concept. Beta casein is phosphorylated at five different serine residues: S-15, S-17, S-18, S-19, and S-36. Digestion with sequencing grade trypsin hydrolyzes at $\mathrm{C}$-terminal lysine (K) and arginine (R) residues. The Q1 MS spectrum of beta casein digest after selective extraction followed by MOI desorption in figure 4 clearly shows that three phosphopeptides at $\mathrm{m} / \mathrm{z}=687.5(\mathrm{P} 1), 742.8(\mathrm{P} 2), 780.7(\mathrm{P} 3)$ are predominantly present, while the peak at $\mathrm{m} / \mathrm{z}=748.8(\mathrm{P} 2 * \mathrm{Na})$ is the $\mathrm{Na}$ adduct of peptide P2. The peak at $\mathrm{m} / \mathrm{z}=830.45(\mathrm{NP})$ is a non-phosphorylated peptide present in abundance due to the presence of hydrophobic residues, which can be eliminated by optimizing the washing method. We were unable to identify another prominent peak at $\mathrm{m} / \mathrm{z}=685.5$ based on Q1 data; a cursory examination would suggest that this peak belongs to a modified form of the phosphopeptide P1, although this hypothesis was not tested at this time. Detailed information can be found in table S4. Other peaks presented in the spectrum were very low in intensity and not identified because were out of the aim of this work. The tryptic digest of beta casein should produce more than 25 different peptides, three of these being phosphopeptides. In this experiment, equilibrium was achieved for most of the detected phosphopeptides within 15 minutes without application of forced convection. Notably, the particles employed in this experiment were in the low micro-size; thus, the kinetics of diffusion can still be significantly enhanced by decreasing the size of the particles. In order to establish the feasibility of this approach, the developed methodology was cross-validated by nano-LC-qTOF. For this purpose, desorption of validation samples was carried out in a vial containing $50 \mu \mathrm{L}$ of desorption solvent $\left(80 \% \mathrm{ACN}+20 \% \mathrm{H}_{2} \mathrm{O}+0.1 \% \mathrm{FA}\right)$ for $60 \mathrm{~min}$ at 1500 $\mathrm{rpm}$. Detailed information regarding the protocol used for the validation procedure is available in supplementary information section 5. The chromatographic region where the characteristic phosphopeptides appear, and the mass spectrum of that region are presented in Figures S6A and S6B, respectively. Table S5 provides a summary of phosphopeptides detected by this gold standard methodology. Both approaches were able to characterize P1, P2, and P3, while P4 was only detected by the separative technique. Figures S7-A and B demonstrate the selectivity of the droplet dispersive microextraction approach in comparison to the validation technique; as can be seen, the inherent lack of cleanup and preconcentration that characterizes the validation technique yielded a great number of peptides and nonspecific 
interferences in comparison to the presented method. As a main feature, the time of analysis for the herein presented method is about 16 min including extraction, while the HR-LC method necessitated an analysis period of about $50 \mathrm{~min}$, excluding the time needed for column equilibration. It should be emphasized that this work represents just a proof of concept, and future work will focus on the development of a coupling interface between dispersive extraction and high-resolution mass spectrometry. Overall, the methodology demonstrated great potential in directly coupling dispersive selective extraction to mass spectrometry via MOI-MS for major phosphopeptides characterization directly from protein digests.

\section{CONCLUSIONS}

Two main technological advances were presented as part of this work. The first advance concerns the development of an MOIbased technology capable of generating an isolated droplet with a volume below $5 \mu \mathrm{L}$ that can be used for desorption of collected particles, or any other kind of surface. Secondly, this work presents a significant advance in dispersive droplet extraction methodology via the generation of a protocol that efficiently disperses micro- or nanoparticles in a sample, then quickly collects particles in a 3D-printed holder containing an embedded magnet. Beyond the simplification of the analytical workflow of magnetic dispersive extraction, the methodology also enabled significant reductions in sample consumption, a dramatic minimization in the number of nanoparticles employed per sample (20-200-folds), and a twofold decrease in the sample-sorbent ratio. Hence, the herein introduced work fills a present gap in the research literature by presenting a first successful instance of direct coupling of magnetic dispersive extraction to MS. As proof of concept, two applications were successfully developed. The first application had as focus the determination of a group of prohibited substances (cocaine, methadone, sertraline, propranolol and fentanyl) from PBS and human urine. In addition to the satisfactory precision $(\leq 20 \%)$ and accuracy (80-120\%) values yielded by the method, the LOQs achieved for both matrices were always within 0.3 and $5 \mathrm{ng} \cdot \mathrm{mL}^{-}$ ${ }^{1}$, meeting stated MRPL values. The whole analytical process was completed in less than a minute. The second application sought to establish a method for selective extraction of phosphopeptides from a $\beta$-casein tryptic digest by employment of Ti-IMAC magnetic microparticles. In this line, several characteristic phosphopeptides were identified. The method was cross-validated by nano-LC-qTOF, using the same samples employed for dispersive SPME coupled to MOI-MS. For the first time, the feasibility of direct hyphenation of magnetic dispersive SPME with MS was demonstrated. This work demonstrated the capability of the technology and methodologies herein presented to analyze small molecules as well as heavier biomolecules such as phosphopeptides while concurrently minimizing sample and sorbent consumption, significantly decreasing time of analysis, and imparting comparable method sensitivity in comparison to traditional methods. Fifteen minutes were required to reach equilibrium, but this extraction time can be shortened, if agitation is applied not only to disperse the particles, but also throughout the extraction process. Also, the use of higher sample-sorbent ratios would result in higher enrichment and higher sensitivity. ${ }^{30}$ Future work will also be directed at the hyphenation of magnetic dispersive biocompatible SPME to high resolution mass spectrometry (HRMS) so as to develop methodologies for fast screening of signature peptides and phosphopeptides of different proteins from complex matrices.

\section{ASSOCIATED CONTENT}

\section{Supporting Information}

The supporting information provided for this work contains the experimental protocols employed for $\mathrm{Fe}_{2} \mathrm{O}_{3}-\mathrm{C}_{18}$ synthesis as well as its characterization. The supporting information also offers details regarding sample preparation of biological matrices, protein digestion, and the LC-HRMS method and results for phosphopeptide determination. Additional experimental information about magnetic dispersive extraction is also presented. Details regarding MS parameters used to quantify each compound, as well as accuracy and repeatability values from different matrices are also available. Finally, calibration curves and validation points for PBS extractions are offered.

\section{AUTHOR INFORMATION}

\section{Corresponding Author}

* Phone: +1 (519) 888 4641; Fax: +1 (519) 7460435.

E-mail: janusz@uwaterloo.ca

\section{Author Contributions}

${ }^{\dagger}$ These authors have contributed equally.

\section{Current affiliation}

¥ Instituto de Investigación e Ingeniería Ambiental (3iA), Universidad Nacional de San Martín (UNSAM), San Martín, Buenos Aires, Argentina.

Notes

The authors declare no competing financial interest.

\section{ACKNOWLEDGMENTS}

The authors are grateful to SCIEX and the Natural Sciences and Engineering Research Council (NSERC) of Canada for the financial support provided through the Industrial Research Chair program. Finally, we would like to express our sincere gratitude to Hiruy Haile and the Science Technical Services at the University of Waterloo for their technical support and collaboration during the construction and improvement of the MOI interface.

\section{REFERENCES}

(1) Monge, M. E.; Harris, G. A.; Dwivedi, P.; Fernández, F. M. Mass Spectrometry: Recent Advances in Direct Open Air Surface Sampling/Ionization. Chem. Rev. 2013, 113 (4), 2269-2308.

(2) Venter, A. R.; Douglass, K. A.; Shelley, J. T.; Hasman, G.; Honarvar, E. Mechanisms of Real-Time, Proximal Sample Processing during Ambient Ionization Mass Spectrometry. Anal. Chem. 2014, 86 (1), 233-249.

(3) Takáts, Z.; Wiseman, J. M.; Gologan, B.; Cooks, R. G. Mass Spectrometry Sampling under Ambient Conditions with Desorption Electrospray Ionization. Science (80-. ). 2004, 306 (5695), 471-473.

(4) Cody, R. B.; Larame, J. A. Versatile New Ion Source for the Analysis of Materials in Open Air under Ambient Conditions. Anal. Chem. 2005, 77 (8), 2297-2302.

(5) Wang, H.; Liu, J.; Graham Cooks, R.; Ouyang, Z. Paper Spray for Direct Analysis of Complex Mixtures Using Mass Spectrometry. Angew. Chemie - Int. Ed. 2010, 49 (5), 877-880.

(6) Kuo, C. P.; Shiea, J. Application of Direct Electrospray Probe to Analyze Biological Compounds and to Couple to Solid-Phase Microextraction to Detect Trace Surfactants in Aqueous Solution. Anal. Chem. 1999, 71 (19), 4413-4417.

(7) Ferreira, C. R.; Yannell, K. E.; Jarmusch, A. K.; Pirro, V.; Ouyang, Z.; Cooks, R. G. Ambient Ionization Mass Spectrometry for Point-of-Care Diagnostics and Other Clinical Measurements. Clin. Chem. 2015, 62 (1), 99-110.

(8) Pirro, V.; Alfaro, C. M.; Jarmusch, A. K.; Hattab, E. M.; CohenGadol, A. A.; Cooks, R. G. Intraoperative Assessment of Tumor Margins during Glioma Resection by Desorption Electrospray Ionization-Mass Spectrometry. Proc. Natl. Acad. Sci. 2017, 114 (26), 6700-6705.

(9) Crawford, E.; Musselman, B. Evaluating a Direct Swabbing Method for Screening Pesticides on Fruit and Vegetable Surfaces 
Using Direct Analysis in Real Time (DART) Coupled to an Exactive Benchtop Orbitrap Mass Spectrometer. Anal. Bioanal. Chem. 2012, 403 (10), 2807-2812.

(10) Wang, Q.; Zheng, Y.; Zhang, X.; Han, X.; Wang, T.; Zhang, Z. A Silica Coated Paper Substrate: Development and Its Application in Paper Spray Mass Spectrometry for Rapid Analysis of Pesticides in Milk. Analyst 2015, 140 (23), 80488056.

(11) Pavlovich, M. J.; Musselman, B.; Hall, A. B. Direct Analysis in Real Time-Mass Spectrometry (DART-MS) in Forensic and Security Applications. Mass Spectrom. Rev. 2018, 37, 171-187.

(12) Costa, C.; Webb, R.; Palitsin, V.; Ismail, M.; De Puit, M.; Atkinson, S.; Bailey, M. J. Rapid, Secure Drug Testing Using Fingerprint Development and Paper Spray Mass Spectrometry. Clin. Chem. 2017, 63 (11), 1745-1752.

(13) Ren, Y.; McLuckey, M. N.; Liu, J.; Ouyang, Z. Direct Mass Spectrometry Analysis of Biofluid Samples Using Slug-Flow Microextraction Nano-Electrospray Ionization. Angew. Chemie Int. Ed. 2014, 53 (51), 14124-14127.

(14) Eikel, D.; Vavrek, M.; Smith, S.; Bason, C.; Yeh, S.; Korfmacher, W. A.; Henion, J. D. Liquid Extraction Surface Analysis Mass Spectrometry (LESA-MS) as a Novel Profiling Tool for Drug Distribution and Metabolism Analysis: The Terfenadine Example. Rapid Commun. Mass Spectrom. 2011, 25 (23), 35873596.

(15) Eikel, D.; Henion, J. D. Liquid Extraction Surface Analysis (LESA): A New Mass Spectrometry-Based Technique for Ambient Surface Profiling. In Mass Spectrometry for Drug Discovery and Drug Development; John Wiley \& Sons, Inc.: Hoboken, NJ, USA, 2013; pp 221-238.

(16) Van Berkel, G. J.; Sanchez, A. D.; Quirke, J. M. E. Thin-Layer Chromatography and Electrospray Mass Spectrometry Coupled Using a Surface Sampling Probe. Anal. Chem. 2002, 74 (24), 6216-6223.

(17) Cahill, J. F.; Kertesz, V.; Weiskittel, T. M.; Vavrek, M.; Freddo, C.; Van Berkel, G. J. Online, Absolute Quantitation of Propranolol from Spatially Distinct 20- and 40-Mm Dissections of Brain, Liver, and Kidney Thin Tissue Sections by Laser Microdissection-Liquid Vortex Capture-Mass Spectrometry. Anal. Chem. 2016, 88 (11), 6026-6034.

(18) Li, A.; Paine, M. R. L.; Zambrzycki, S.; Stryffeler, R. B.; Wu, J.; Bouza, M.; Huckaby, J.; Chang, C.-Y.; Kumar, M.; Mukhija, P.; et al. Robotic Surface Analysis Mass Spectrometry (RoSA-MS) of Three-Dimensional Objects. Anal. Chem. 2018, 90 (6), 39813986.

(19) Neu, V.; Steiner, R.; Müller, S.; Fattinger, C.; Zenobi, R. Development and Characterization of a Capillary Gap Sampler as New Microfluidic Device for Fast and Direct Analysis of Low Sample Amounts by ESI-MS. Anal. Chem. 2013, 85 (9), 46284635.

(20) Reyes-Garcés, N.; Gionfriddo, E.; Gómez-Ríos, G. A.; Alam, M. N.; Boyacl, E.; Bojko, B.; Singh, V.; Grandy, J.; Pawliszyn, J. Advances in Solid Phase Microextraction and Perspective on Future Directions. Anal. Chem. 2018, 90 (1), 302-360.

(21) Piri-Moghadam, H.; Alam, M. N.; Pawliszyn, J. Review of Geometries and Coating Materials in Solid Phase Microextraction: Opportunities, Limitations, and Future Perspectives. Anal. Chim. Acta 2017.

(22) Miller, V. P. SPE-MS Analysis of Absorption, Distribution, Metabolism and Excretion Assays: A Tool to Increase Throughput and Steamline Workflow. Bioanalysis 2012, 4 (9), 1111-1121.

(23) Dolder, P. C.; Liechti, M. E.; Rentsch, K. M. Development and Validation of a Rapid Turboflow LC-MS/MS Method for the Quantification of LSD and 2-Oxo-3-Hydroxy LSD in Serum and Urine Samples of Emergency Toxicological Cases. Anal. Bioanal. Chem. 2015, 407 (6), 1577-1584.

(24) Gómez-Ríos, G. A.; Pawliszyn, J. Development of Coated Blade Spray Ionization Mass Spectrometry for the Quantitation of Target Analytes Present in Complex Matrices. Angew. Chemie Int. Ed. 2014, 53 (52), 14503-14507.

(25) Gómez-Ríos, G. A.; Pawliszyn, J. Solid Phase Microextraction (SPME)-Transmission Mode (TM) Pushes down Detection Limits in Direct Analysis in Real Time (DART). Chem. Commun. (Camb). 2014, 50 (85), 12937-12940.

(26) Piri-Moghadam, H.; Lendor, S.; Pawliszyn, J. Development of a
Biocompatible In-Tube Solid-Phase Microextraction Device: A Sensitive Approach for Direct Analysis of Single Drops of Complex Matrixes. Anal. Chem. 2016, 88 (24), 12188-12195. Tascon, M.; Alam, M. N.; Gómez-Ríos, G. A.; Pawliszyn, J. Development of a Microfluidic Open Interface with Flow Isolated Desorption Volume for the Direct Coupling of SPME Devices to Mass Spectrometry. Anal. Chem. 2018, 90 (4), 26312638.

(28) Tascon, M.; Gómez-Ríos, G. A.; Reyes-Garcés, N.; Poole, J.; Boyac1, E.; Pawliszyn, J. High-Throughput Screening and Quantitation of Target Compounds in Biofluids by Coated Blade Spray-Mass Spectrometry. Anal. Chem. 2017, 89 (16), 84218428.

(29) Gómez-Ríos, G. A.; Gionfriddo, E.; Poole, J.; Pawliszyn, J. Ultrafast Screening and Quantitation of Pesticides in Food and Environmental Matrices by Solid-Phase MicroextractionTransmission Mode (SPME-TM) and Direct Analysis in Real Time (DART). Anal. Chem. 2017, 89 (13).

(30) Alam, M. N.; Nazdrajić, E.; Singh, V.; Tascon, M.; Pawliszyn, J. Effect of Transport Parameters and Device Geometry on Extraction Kinetics and Efficiency in Direct Immersion SolidPhase Microextraction. Anal. Chem. 2018, 90 (19), 1154811555.

(31) Pawliszyn, J. Handbook of Solid-Phase Microextraction; Industry Press: Beijing, 2009.

(32) Alam, M. N.; Pawliszyn, J. Effect of Binding Components in Complex Sample Matrices on Recovery in Direct Immersion Solid-Phase Microextraction: Friends or Foe? Anal. Chem. 2018, 90 (4), 2430-2433.

(33) Wierucka, M.; Biziuk, M. Application of Magnetic Nanoparticles for Magnetic Solid-Phase Extraction in Preparing Biological, Environmental and Food Samples. TrAC Trends Anal. Chem. 2014, 59, 50-58.

(34) Plotka-Wasylka, J.; Szczepanska, N.; de la Guardia, M.; Namiesnik, J. Modern Trends in Solid Phase Extraction: New Sorbent Media. TrAC - Trends Anal. Chem. 2016, 77, 23-43.

(35) Chen, D.; Zheng, H. B.; Huang, Y. Q.; Hu, Y. N.; Yu, Q. W.; Yuan, B. F.; Feng, Y. Q. Magnetic Solid Phase Extraction Coupled with Desorption Corona Beam Ionization-Mass Spectrometry for Rapid Analysis of Antidepressants in Human Body Fluids. Analyst 2015, 140 (16), 5662-5670.

(36) Van Berkel, G. J.; Kertesz, V. An Open Port Sampling Interface for Liquid Introduction Atmospheric Pressure Ionization Mass Spectrometry. Rapid Commun. Mass Spectrom. 2015, 29 (19), $1749-1756$.

(37) Gomez-ríos, G. A.; Liu, C.; Tascon, M.; Reyes-Garces, N.; Arnold, D. W.; Covey, T. R.; Pawliszyn, J. Open Port Probe Sampling Interface for the Direct Coupling of Biocompatible Solid-Phase Microextraction to Atmospheric Pressure Ionization Mass Spectrometry. Anal. Chem. 2017, 89 (7), 3805-3809.

(38) Manicke, N. E.; Bills, B. J.; Zhang, C. Analysis of Biofluids by Paper Spray MS: Advances and Challenges. Bioanalysis 2016, 8 (6), 589-606.

(39) Vasconcelos, I.; Fernandes, C. Magnetic Solid Phase Extraction for Determination of Drugs in Biological Matrices. TrAC Trends Anal. Chem. 2017, 89, 41-52.

(40) Gómez-Ríos, G. A.; Tascon, M.; Reyes-Garcés, N.; Poole, J.; Boyac1, E.; Pawliszyn, J. Quantitative Analysis of Biofluid Spots by Coated Blade Spray Mass Spectrometry, a New Approach to Rapid Screening. Sci. Rep. 2017, 7, 16104.

(41) Mirabelli, M. F.; Wolf, J.-C.; Zenobi, R. Direct Coupling of Solid-Phase Microextraction with Mass Spectrometry: Sub-Pg/g Sensitivity Achieved Using a Dielectric Barrier Discharge Ionization Source. Anal. Chem. 2016, 88 (14), 7252-7258.

(42) Gaissmaier, T.; Siebenhaar, M.; Todorova, V.; Hüllen, V.; Hopf, C.; Dartois, V.; McKay, G.; Miller, K. J.; Patnaik, R. N.; Powell, M. L.; et al. Therapeutic Drug Monitoring in Dried Blood Spots Using Liquid Microjunction Surface Sampling and High Resolution Mass Spectrometry. Analyst 2016, 141 (3), 892-901.

(43) Wang, G. N.; Wu, N. P.; He, X.; Zhang, H. C.; Liu, J.; Wang, J. P. Magnetic Graphene Dispersive Solid Phase Extraction-Ultra Performance Liquid Chromatography Tandem Mass Spectrometry for Determination of $\beta$-Agonists in Urine. $J$. Chromatogr. B Anal. Technol. Biomed. Life Sci. 2017, 1067, 1824. 


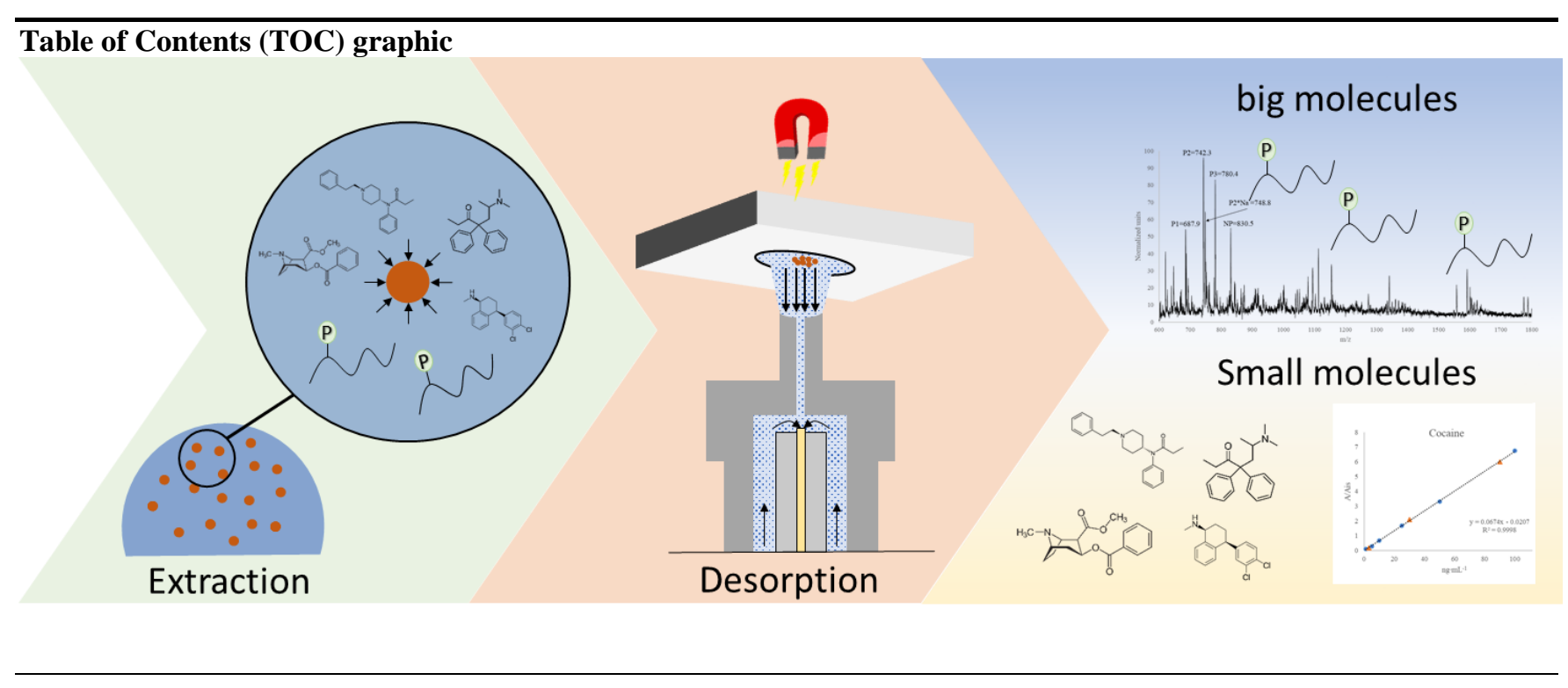

